\title{
Emotions Evoked by Traditional Chinese Herbs for Cosmeceuticals
}

\author{
Nan QIE, Pei-Luen Patrick RAU and Jiamin DENG \\ Tsinghua University, Beijing, China
}

\begin{abstract}
Odor directly evokes emotions, and plays an important role in purchase decision of cosmetics. This research studied emotions evoked by 10 traditional Chinese herb odors. Fifty Chinese and fifty Korean female students participated in the experiment. Each participant smelled all the odors and filled in Emotion and Odor Scales to report their emotions. Participants were also asked to choose one among three cloths, which can best match the odor. This part was involved to study olfaction-touch interaction. Chinese participants rated significantly higher than Korean participants in all the positive emotional dimensions, and in the arousal dimension. Participants from both China and Korea preferred Sweet Osmanthus and Raspberry, while disliked Camphor and Musk. Participants tended to relate more pleasant odors to softer and smoother touch.
\end{abstract}

Keywords: Odor perception, Cultural difference, Olfaction-touch interaction

\section{INTRODUCTION}

Using herbs instead of artificial compositions in cosmetic products is thought to be safer and more natural. Along with this trend, the value of traditional Chinese medicine is again treasured today. In the field of Chinese medicine, a large amount of herbs of various functions are studied and used. Applying Chinese medicine in cosmetic products can be a promising direction for product design. Odor, which is one of most important aspects in cosmetic product design, should be specially studied for cosmeceuticals using Chinese medicine.

Cultural differences on odor-evoked emotions have been proved in previous studies. These differences are mainly from odor familiarity and symbolization. People tend to think more positive of odors that are more familiar to them, and those that have positive meanings in the culture $[8,9]$. The results of these studies are usually obtained from two cultural groups that are commonly thought to have large diversity, for example, from Switzerland, the United Kingdom and Singapore [5], or from France, Vietnam and America [2]. There are hardly any researches on differences in odor-evoked emotions from similar cultures. However, along with market segmentation, more specific studies should be conducted. Odor perception between two cultures that are not very different can also exist. Both China and Korea belong to Eastern Asia cultural group and both these two cultures have their own traditional herbs. In this study the differences in perception of Chinese herb odors among Chinese and Korean participants are studied, to provide a guidance for cosmeceuticals design in both these two cultures.
Design of a cosmetic product should consider many aspects, including function, color, odor, shape of the package and even appearance of the model showing in advertisement. Consistency among these aspects can help improve consumers' experience with the product and enhance purchasing [10]. As a result, there have been researchers focusing on synesthesia, which is a neurological phenomenon in which stimulation of one sensory or cognitive pathway leads to automatic, involuntary experiences in a second sensory or cognitive pathway [3]. Proofs have been found in relationship between olfactory and vision $[1,4,7]$. Besides olfactory and vision, touch is also very important in cosmetic product design, in both the product itself and its package. However, no existent research in synesthesia between olfactory and touch has been found. This study is going to study the relationship between olfactory and touch for the first time ever, in order to help with cosmeceutical design.

Popularity of cosmeceuticals provides opportunity for traditional Chinese medicine to be used in cosmetic products. This research aims to study how odors of 10 well-selected traditional Chinese herbs are perceived by participants from China and Korea. Besides, this research will for the first time explore the relationship between olfactory and touch. Results of this research can provide meaningful guidance for future cosmeceutical design.

\section{METHOD}

\subsection{Pilot study}

We conducted a pilot study of 20 Chinese and 20 Korean female participants. In the pilot study we used 
both (1) subjective rated scales (the Emotion and Odor Scales, EOS for Chinese) and (2) physiological measures including skin conductance ( $\mathrm{SC}$, in $\mu \mathrm{S}$ ), electromyography on corrugator muscles (CEMG, in $\mu \mathrm{V}$ ) and zygomatic muscles (ZEMG, in $\mu \mathrm{V}$ ) to measure the odor-evoked emotions. The subjective rated scales worked well while the physiological measures failed to provide any convictive results. So we deleted physiological measurements from experiment design in the formal experiment.

\subsection{Participant}

Fifty Chinese and fifty Koreans participated. All the participants were students of Tsinghua University, with ages ranging from 17 to 24 (mean $=21.2, \mathrm{SD}=1.6$ ). Plenty of previous studies have shown evidence of gender difference on odor perception. Considering the limited sample size and the main target consumers of cosmeceuticals, we only recruited female to participate.

\subsection{Experiment Design}

A mixed experiment design was conducted. Every participant from either China or Korea should smell all the 10 odors. Here culture was a between-subject factor and odor was a within-subject factor.

In order to get rid of the influence of odor presenting order, a Latin Square Design was used. The participants were divided into 10 groups. Within each group there were 5 Chinese and 5 Koreans.

\subsection{Subjective emotional scales}

Emotion and Odor Scales (EOS) has been widely used to measure odor-evoked emotions. Researchers have found that people from different cultures tend to use different verbalization to describe emotions evoked by odors, so personalized scales for different cultures are necessary. In this study, an EOS specially developed for Chinese was used [6]. Both Chinses and Korean participants used the same scales. Because (1) there haven't been any EOS specially developed for Koreans and (2) all our Korean participants were overseas students in China, it was acceptable to use the Chinese EOS for all the participants. The original questionnaire was English and was back-translated into Chinese. Chinese participants all used the Chinese version, and Korean participants can choose to use either the Chinese version or the English version.

The scales used in this study had in total 26 items, belonging to 7 emotional dimensions including HW (Happiness / Well-being), DI (Disgust / Irritation), SP (Smoothing / Peacefulness), DE (Desire), AR (Arousal), EN (Energy) and ME (Melancholy). Among these 7 dimensions, ME was specially added in Chinses EOS, compared to the original EOS. Seven-point Likert scales were used in this questionnaire, the participants were asked to rate from 1 (not at all) to 7 (totally agree) for each emotion description.

\subsection{Touch measurement and related questionnaire}

In this study, touch was measured by asking the participant to choose one cloth from three cloths provided. The three cloths were made by linen (No.1), cotton (No.2) and rayon (No.3), respectively. The clothes were all $10 \mathrm{~cm} * 10 \mathrm{~cm}$ large, white in color, and cannot be distinguished by vision. Only touch of them was different. No.1 was the hardest and roughest, while No.3 was the softest and smoothest.

In the post-smelling questionnaire, the participants were asked to choose one cloth whose touch can best describe the odor. And they were also asked to rate the odor on pleasure, irritation and intensity dimension according to their own feelings, from 1 (not at all) to 7 (totally agree). The cloth choice and these basic odor features can provide insight into the relationship between olfactory and touch.

\subsection{Odor Stimuli}

Ten odors of traditional Chinese herbs were chosen to be stimuli (Table 1). Among these 10 odors, No. 1 and No. 5 were flowers. No. 6 was fruit. No. 9 and No. 10 were traditional Chinses functional perfumes made up by herbs. All these stimuli were used in traditional Chinese medicine, or have been used as spice. Chinese people were familiar with their smells, and were familiar with the situations where each of them may appear.

The stimuli were liquid placed in non-transparent plastic bottles with the same appearance. The participants can't distinguish them by vision. The stimuli were checked and replaced frequently, in order to get rid of the bias caused by volatilization.

Table 1: Odors used in the experiment

\begin{tabular}{|c|l|}
\hline No. & \multicolumn{1}{|c|}{ Odor } \\
\hline 1 & Sweet osmanthus \\
\hline 2 & Camphor \\
\hline 3 & Musk \\
\hline 4 & Sandalwood \\
\hline 5 & Calendula \\
\hline 6 & Raspberry \\
\hline 7 & Bamboo \\
\hline 8 & Green tea \\
\hline 9 & Repellent floral water \\
\hline 10 & Bezoar floral water \\
\hline
\end{tabular}




\subsection{Experiment procedure}

The experiment was conducted in a lab of 25 centigrade and with no ventilation during experiment. The constant temperature can ensure that every participant feel the same intensity of smelling. No ventilation can ensure control of environmental smell disturbance. The experiment lasted about $40 \mathrm{~min}$ for each participant.

Each participant was welcomed to the lab, sit in a chair, turned off the cell phone and filled in an informed content. She was introduced about the instruments to be used, and about the whole experiment procedure.

Then came to the experiment section. This section included 10 cycles. During each cycle, an odor would be tested. At the beginning of a cycle, the participant was asked to just sit there quietly and keep calm for $20 \mathrm{~s}$. Then the experimenter would put one bottle of odor about $2 \mathrm{~cm}$ below the participant's nose, and the participant was asked to sit still and just smell for another $20 \mathrm{~s}$. After smelling the odor, the participant was asked to fill in a questionnaire according to the odor they just smelled. This questionnaire included two parts, one was the emotional scales and one was the touch-olfactory part. At the end of each section, the participant smelled cocoa powder to clean their memory about the previous odor. After that they were asked to calm down and came to the next cycle.

After 10 cycles had been finished, the participant had the opportunity to comment about the previous odors freely, and the experimenter would tell the participant the content in each bottle. Each participant got 40 RMB as reward.

HW

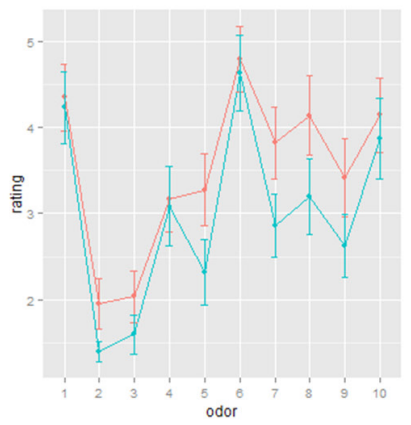

AR

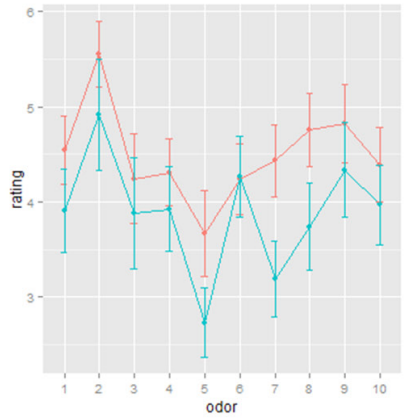

DI

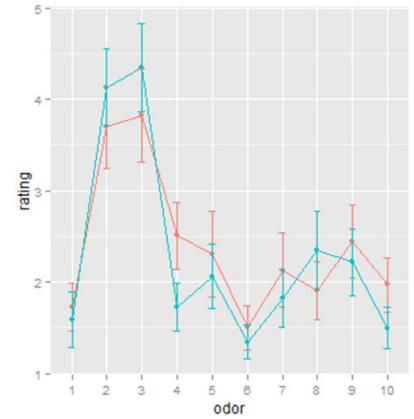

EN

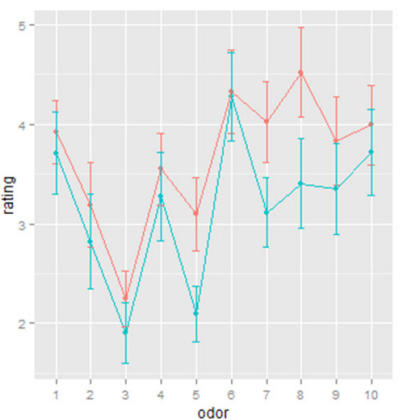

\section{RESULT}

\subsection{EOS}

Cronbach's alphas were calculated to test the inner consistency of emotional scales in each emotional dimension. All the items in every emotional dimension had good inner consistency, with Cronbach's alpha larger than 0.70.

The descriptive statistics of ratings on each emotional dimension, for each odor and for each culture were calculated and summarized in Table 2 and Figure 1. From

Table 2: Descriptive statistics of EOS

\begin{tabular}{|c|l|c|c|c|c|c|c|c|}
\hline \multicolumn{2}{|c|}{ Odor } & HW & DI & SP & DE & AR & EN & ME \\
\hline \multirow{2}{*}{1} & Mean & 4.29 & 1.66 & 4.40 & 4.12 & 4.24 & 3.82 & 2.05 \\
\cline { 2 - 9 } & SD & 1.38 & 1.98 & 1.60 & 1.51 & 1.39 & 1.25 & 1.05 \\
\hline \multirow{2}{*}{2} & Mean & 1.69 & 3.90 & 1.63 & 1.55 & 5.24 & 3.01 & 3.14 \\
\cline { 2 - 9 } & SD & 0.84 & 1.52 & 1.05 & 0.85 & 1.64 & 1.55 & 1.48 \\
\hline \multirow{3}{*}{3} & Mean & 1.82 & 4.08 & 2.07 & 1.66 & 4.07 & 2.08 & 3.59 \\
\cline { 2 - 9 } & SD & 0.94 & 1.72 & 1.41 & 0.96 & 1.81 & 1.01 & 1.51 \\
\hline \multirow{3}{*}{4} & Mean & 3.13 & 2.13 & 3.56 & 2.73 & 4.13 & 3.42 & 2.78 \\
\cline { 2 - 9 } & SD & 1.44 & 1.18 & 1.63 & 1.40 & 1.37 & 1.39 & 1.23 \\
\hline \multirow{2}{*}{5} & Mean & 2.82 & 2.19 & 3.58 & 2.74 & 3.22 & 2.62 & 2.87 \\
\cline { 2 - 9 } & SD & 1.45 & 1.44 & 1.82 & 1.52 & 1.50 & 1.23 & 1.52 \\
\hline \multirow{3}{*}{6} & Mean & 4.71 & 1.43 & 4.56 & 4.42 & 4.25 & 4.30 & 1.97 \\
\cline { 2 - 8 } & SD & 1.40 & 1.75 & 1.48 & 1.38 & 1.36 & 1.47 & 0.94 \\
\hline \multirow{2}{*}{7} & Mean & 3.36 & 1.98 & 4.22 & 3.18 & 3.83 & 3.59 & 2.69 \\
\cline { 2 - 8 } & SD & 1.44 & 1.27 & 1.67 & 1.48 & 1.48 & 1.39 & 1.43 \\
\hline \multirow{2}{*}{8} & Mean & 3.69 & 2.12 & 3.18 & 3.52 & 4.27 & 3.98 & 2.37 \\
\cline { 2 - 8 } & SD & 1.62 & 1.28 & 1.72 & 1.73 & 1.53 & 1.64 & 1.31 \\
\hline \multirow{2}{*}{9} & Mean & 3.04 & 2.33 & 3.09 & 3.00 & 4.59 & 3.60 & 2.80 \\
\cline { 2 - 8 } & SD & 1.48 & 1.32 & 1.69 & 1.58 & 1.57 & 1.55 & 1.52 \\
\hline \multirow{2}{*}{10} & Mean & 4.01 & 1.74 & 4.09 & 3.86 & 4.19 & 3.86 & 2.32 \\
\cline { 2 - 8 } & SD & 1.55 & 0.96 & 1.65 & 1.68 & 1.39 & 1.44 & 1.16 \\
\hline
\end{tabular}

$\mathrm{SP}$

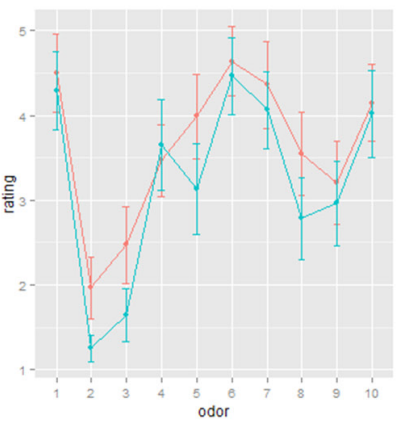

ME

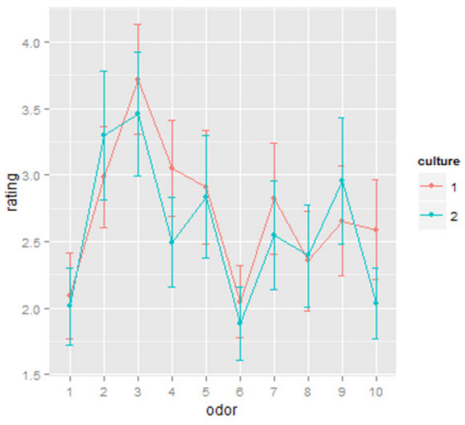

$\mathrm{DE}$

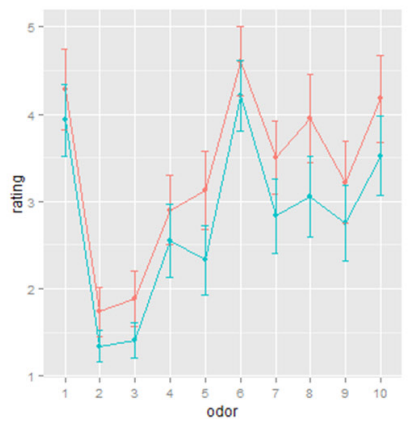

Figure 1: Mean rating plots in seven emotional dimensions (culture 1-China; culture 2-Korea) 
the plots we found that in all the positive emotional dimensions including HW, SP, DE, and EN, Chinese participants gave higher ratings for almost all the odors than Korean participants. For AR dimension, Chinese participants gave higher ratings for all the odors than Korean participants.

Because most of the data subsets can not pass normality test, non-parametric tests were conducted to test the effect of culture and odor, for each emotional dimension respectively. The effect of culture on ratings was tested by Wilcox test (Table 3). The effect of culture was significant in all the positive emotional dimensions including HW, SP, DE, and EN, and was significant in AR dimension. In all these dimensions Chinese participants rated significantly higher than Korean participants. This indicated that Chinese participants showed more positive emotions towards the 10 odors in this experiment, and their arousal rate was higher. The effect of odor was tested by Kruskal-Wallis test (Table 4). The effect of odor was significant in all the seven dimensions. The detailed difference caused by odor should be further checked by post-hoc test.

For effect of odor, we mainly focus on HW, DI and AR dimensions, which can indicate positive emotion, negative emotion, and arousal rate, respectively. We used bonferroni method to modify the p-value in post-hoc tests. Roughly summarizing the results in HW dimension, we can conclude that odor 1,6 and 10 were significantly more pleasant than other odors $(\mathrm{p}<0.001)$; and odor 2

Table 3: Result of Wilcox test: effect of culture on ratings

\begin{tabular}{|c|c|c|}
\hline Dimension & P & W \\
\hline HW & $<\mathbf{0 . 0 0 1}$ & 137010 \\
\hline DI & 0.111 & 121760 \\
\hline SP & $<\mathbf{0 . 0 0 1}$ & 130050 \\
\hline DE & $<\mathbf{0 . 0 0 1}$ & 135820 \\
\hline AR & $<\mathbf{0 . 0 0 1}$ & 141320 \\
\hline EN & $<\mathbf{0 . 0 0 1}$ & 136020 \\
\hline ME & 0.108 & 121860 \\
\hline
\end{tabular}

Table 4: Result of Kruskal-Wallis test: effect of odor on ratings

\begin{tabular}{|c|c|c|}
\hline Dimension & $\mathrm{P}$ & Chi-square \\
\hline HW & $<\mathbf{0 . 0 0 1}$ & 314.8 \\
\hline DI & $<\mathbf{0 . 0 0 1}$ & 267.99 \\
\hline SP & $<\mathbf{0 . 0 0 1}$ & 254.32 \\
\hline DE & $<\mathbf{0 . 0 0 1}$ & 294.33 \\
\hline AR & $<\mathbf{0 . 0 0 1}$ & 88.46 \\
\hline EN & $<\mathbf{0 . 0 0 1}$ & 170.28 \\
\hline ME & $<\mathbf{0 . 0 0 1}$ & 99.29 \\
\hline
\end{tabular}

and 3 were significantly less pleasant than other odors $(\mathrm{p}<0.001)$. In DI dimension, we can conclude that odor 2 and 3 were significantly more unpleasant than all other odors $(p<0.001)$; and odor 6 was significantly less unpleasant than other odors except for odor 1 and odor $10(\mathrm{p}<0.001)$. The results in HW and DI dimensions were consistent. In AR dimension, we can conclude that the emotional arousal rate evoked by odor 5 was significantly lower than other odors $(\mathrm{p}<0.001)$; and the emotional arousal rate evoked by odor 2 was significantly higher than other odors $(\mathrm{p}<0.001)$.

Non-parametric test can not test interaction effect. In order to have a general idea of cultural difference for each odor, Wilcox test was conducted (Table 5). Chinese participants gave significantly higher ratings for odor 2, 3, 5, 7, 8 and 9. Odor 1, 6 and 10 were the three most pleasant rated odors in this experiment, where Chinese and Korean participants showed no significant difference in HW dimension ratings. Odor 2 and 3 were the two most unpleasant rated odors in this experiment. Chinese participants showed more tolerance to them. Although no significant difference showed, Chinese participants gave higher ratings for odor 4 in the two negative emotional dimensions. Chinese participants rated significantly higher than Koreans for odor 5 in all positive emotional dimensions and AR dimension. In all seven dimensions, where significant cultural difference existed, Chinese participants all gave higher ratings than Koreans.

\subsection{Relationship between olfactory and touch}

Main effects plots between cloth choice and subjective rated pleasure, intensity and irritation were graphed separately, and were shown in Figure 2. The plots indicated that participants tended to choose softer and smoother cloth for more pleasant, less irritated odors.

Table 5: P-values of Wilcox test on effect of culture in each odor

\begin{tabular}{|c|c|c|c|c|c|c|c|}
\hline Odor & HW & DI & SP & DE & AR & EN & ME \\
\hline 1 & 0.550 & $\mathbf{0 . 0 7 2}$ & 0.433 & 0.203 & $\mathbf{0 . 0 1 6}$ & 0.373 & 0.849 \\
\hline 2 & $\mathbf{0 . 0 1 5}$ & 0.265 & $<\mathbf{0 . 0 0 1}$ & 0.054 & 0.375 & 0.205 & 0.451 \\
\hline 3 & $\mathbf{0 . 0 2 3}$ & 0.191 & $\mathbf{0 . 0 0 4}$ & $\mathbf{0 . 0 3 6}$ & 0.350 & $\mathbf{0 . 0 3 3}$ & 0.464 \\
\hline 4 & 0.597 & $\mathbf{0 . 0 0 5}$ & 0.617 & 0.146 & 0.178 & 0.297 & $\mathbf{0 . 0 2 5}$ \\
\hline 5 & $<\mathbf{0 . 0 0 1}$ & 0.909 & $\mathbf{0 . 0 2 4}$ & $\mathbf{0 . 0 0 9}$ & $\mathbf{0 . 0 0 3}$ & $<\mathbf{0 . 0 0 1}$ & 0.729 \\
\hline 6 & 0.633 & 0.170 & 0.413 & 0.112 & 0.818 & 0.930 & 0.370 \\
\hline 7 & $<\mathbf{0 . 0 0 1}$ & 0.468 & 0.385 & $\mathbf{0 . 0 2 3}$ & $<\mathbf{0 . 0 0 1}$ & $\mathbf{0 . 0 0 2}$ & 0.351 \\
\hline 8 & $\mathbf{0 . 0 0 3}$ & 0.179 & $\mathbf{0 . 0 2 7}$ & $\mathbf{0 . 0 1 5}$ & $\mathbf{0 . 0 0 1}$ & $\mathbf{0 . 0 0 1}$ & 0.770 \\
\hline 9 & $\mathbf{0 . 0 1 5}$ & 0.560 & 0.470 & 0.181 & 0.156 & 0.177 & 0.405 \\
\hline 10 & 0.301 & $\mathbf{0 . 0 0 8}$ & 0.693 & $\mathbf{0 . 0 3 5}$ & 0.160 & 0.436 & 0.051 \\
\hline
\end{tabular}



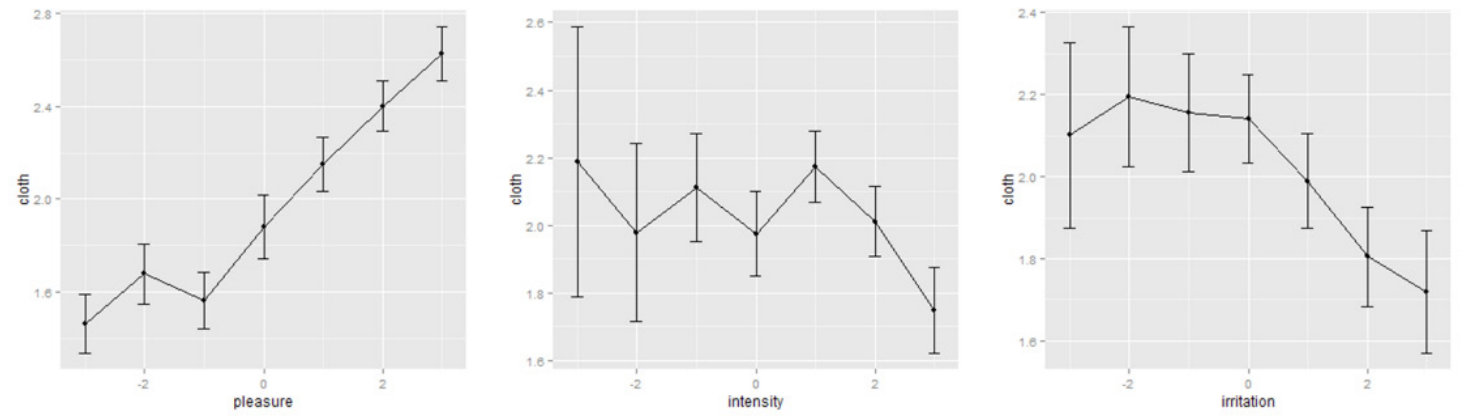

Figure 2: Main effects between cloth choice and subjective $\cdot$ rated pleasure, intensity and irritation

To test this, best subsets regression was conducted to analyze the relationship between olfactory and touch. The result showed that when pleasure was used as independent variable, the model was best, with $\mathrm{P}<0.05$ for both the variable and the model, and $\mathrm{R}$ Square at $28.0 \%$. The regression function was

$$
\text { Cloth }=1.96+0.229 * \text { Pleasure }
$$

The results indicated that there did exist relationship between olfactory and touch. People tended to relate more pleasant odors to softer, smoother touch, while relate less pleasant odors to tougher, harder touch.

\section{DISCUSSION}

This research aims to study the emotions evoked by 10 traditional Chinese herb odors, which we think are promising to be used in cosmeceuticals. Cosmeceuticals are popular both in China and Korea, and have potential to be welcomed by more people around the world, due to their peculiarity in nature and safety.

In this study we compare the emotions evoked by these 10 odors between Chinese and Koreans, and find some differences. Chinese participants give significant higher ratings in all the positive emotional dimensions and in arousal dimension. This is consistent with previous researches stating that people will think odors that are familiar and have positive meanings in their own culture are more pleasant, and show higher arousal rate to these odors. The 10 odors we use in this experiment are all traditional Chinese herb odors. Although some of these odors like raspberry and green tea are also common in Korea, Chinese people are in general more familiar with these 10 odors. China has a long history of herbal medicine. Until now there are still lots of people in China who believe in traditional Chinese medicine, and think traditional herbs as sacred and helpful. So because of the familiarity and symbolization aspects, it is no wonder that Chinese people think more positively and more actively of these 10 odors in the experiment.

Although no significant difference showed, we found that the ratings in the two negative emotional dimensions (DI and ME) given by Chinese participants are also higher than those given by Korean participants. This leads to a confounding whether the cultural difference in those positive emotional dimensions and AR dimension due to familiarity and symbolization issues, or due to the overall tendency for Chinese participants to give higher ratings in questionnaire. From the results of this experiment it is hard to totally exclude the overall bias factor. However, it is reasonable to believe that cultural difference in odor perception does exist, because significant difference showed in positive emotional dimensions and AR dimension but not in negative emotional dimensions.

This research for the first time explores the olfactiontouch interaction phenomenon. According to previous studies of olfaction-vision interaction, and daily experience, we hypothesis that people tend to relate more pleasant, less irritated and less intensive odors to softer and smoother touch. This hypothesis is well demonstrated by the experiment result. The main effect analysis shows that pleasure and irritation may have effect on cloth choosing. The result of best subsets regression finally decides that pleasure can best predict cloth choosing in this experiment. The more pleasant an odor is, the more possible people will relate it to softer and smoother touch. This interesting finding can provide good suggestions for cosmeceuticals product design. Design of the texture and packages should consider the olfaction-touch interaction phenomenon. This can help build a consistent brand image and make the product more impressive.

\section{ACKNOWLEDGEMENTS}

This study was funded by a National Natural Science Foundation China grant 71188001. 


\section{REFERENCES}

1. Barkat, S., Thomas-Danguin, T., Bensafi, M., Rouby, C., and Sicard, G.; Odor and color of cosmetic products: correlations between subjective judgement and autonomous nervous system response. International Journal of Cosmetic Science, 25(6), pp.273-283, 2003.

2. Chrea, C., Valentin, D., Sulmont-Rossé, C., Mai, H. L., Nguyen, D. H., and Abdi, H.; Culture and odor categorization: agreement between cultures depends upon the odors. Food Quality and Preference, 15(7), pp.669-679, 2004.

3. Cytowic, R. E.; Synesthesia: A union of the senses. MIT press, 2002. Retrieved from https://books. google.com/books?hl=zh-CN\&lr=\&id=fl6wX4xzb_ kC\&oi=fnd\&pg=PR7\&dq=Synesthesia:+A+Union+ of +the+Senses $+(2$ nd+edition $) \&$ ots $=$ ii $2 x u K R 5$ ts \&sig =CTuqfDP3h_lneGRpE-bet2j07h8

4. Demattè, M. L., Sanabria, D., and Spence, C.; Olfactory discrimination: when vision matters? Chemical Senses, 34(2), pp.103-109, 2009.

5. Ferdenzi, C., Roberts, S. C., Schirmer, A., Delplanque, S., Cekic, S., Porcherot, C., Cayeux I., Sander D., Grandjean, D.; Variability of affective responses to odors: culture, gender, and olfactory knowledge. Chemical Senses, 38(2), pp.175-186, 2013.

6. Ferdenzi, C., Delplanque, S., Barbosa, P., Court, K., Guinard, J.-X., Guo, T., Roberts S. C., Schirmer A., Porcherot C., Cayeux I., Sander D., and Grandjean D.; Affective semantic space of scents. Towards a universal scale to measure self-reported odor-related feelings. Food Quality and Preference, 30(2), pp.128-138, 2013.
7. Österbauer, R. A., Matthews, P. M., Jenkinson, M., Beckmann, C. F., Hansen, P. C., and Calvert, G. A.; Color of scents: chromatic stimuli modulate odor responses in the human brain. Journal of Neurophysiology, 93(6), pp.3434-3441, 2005.

8. Pangborn, R. M., Guinard, J.-X., and Davis, R. G.; Regional aroma preferences. Food Quality and Preference, 1(1), pp.11-19, 1988.

9. Wysocki, C. J., Pierce, J. D., and Gilbert, A. N.; Geographic, cross-cultural, and individual variation in human olfaction. Smell and Taste in Health and Disease, pp.287-314, 1991.

10. Zarzo, M.; Relevant psychological dimensions in the perceptual space of perfumery odors. Food Quality and Preference, 19(3), pp.315-322, 2008.

Nan QIE (Non-member)

Nan Qie is a Ph.D. student of Institute of Human Factors and Ergonomics, Department of Industrial Engineering, Tsinghua University, China. Her interests are in Cross-cultural Social Psychology, and Kansei Engineering.

Pei-Luen Patrick RAU (Non-member)

Pei-Luen Patrick Rau is a Professor of Institute of Human Factors and Ergonomics, Department of Industrial Engineering, Tsinghua University, China. His interests are Human-Computer Interaction, User Experience, and Cross-Cultural Design.

\section{Jiamin DENG (Non-member)}

Jiamin Deng is a Graduate student of Institute of Engineering Systems, Department of Industrial Engineering, Tsinghua University, China. Her interests are Quality Management and System Support. 